Cytogenet Genome Res. 1975;15:281

\title{
Data on cytogenetic registers
}

The International Advisory Committee on Cytogenetic Registers to the Standing Committee on Standardization in Human Cytogenetics was charged with the responsibility of compiling, updating, and publishing a list of cytogenetic registers, together with summaries of aims, objectives, and key elements. The Committee has developed a form for the collection of such data and invites individuals who operate cytogenetic registers (either in isolation or as part of a larger register system) to write for these data forms as soon as possible to Dr. James R. Miller, Department of Medical Genetics, The University of British Columbia, Vancouver, B.C., V6T 1W5 (Canada). 